\title{
Editorial
}

\section{Testing, Measurement, and Characterization of Nanomaterials}

\author{
Bei Peng, ${ }^{1}$ Changhong Ke, ${ }^{2}$ Yaling Liu, ${ }^{3}$ and Yong $\mathrm{Zhu}^{4}$ \\ ${ }^{1}$ School of Mechatronics Engineering, University of Electronic Science and Technology of China, Chengdu, Sichuan 611731, China \\ ${ }^{2}$ Department of Mechanical Engineering, Binghamton University, State University of New York (SUNY), Binghamton, \\ NY 13902-6000, USA \\ ${ }^{3}$ Department of Mechanical Engineering and Mechanics, Lehigh University, PA 18015, USA \\ ${ }^{4}$ Department of Mechanical and Aerospace Engineering, North Carolina State University, Raleigh, NC 27695-7910, USA
}

Correspondence should be addressed to Bei Peng; beipeng@uestc.edu.cn

Received 6 July 2015; Accepted 6 July 2015

Copyright (C) 2015 Bei Peng et al. This is an open access article distributed under the Creative Commons Attribution License, which permits unrestricted use, distribution, and reproduction in any medium, provided the original work is properly cited.

Materials and structures at the nanoscale often have unique mechanical, electronic, or optical properties. With emergence of various novel nanoscale materials and structures over the past decades, traditional testing and characterization techniques may not be suitable any more especially when structures are formed with sizes comparable to any of many possible length scales. In this special issue, original research articles were selected which cover the mechanical, electrical, thermal, optical, and biological properties of a broad range of nanomaterials. We hope the special issue will stimulate finding of new phenomena and properties and developing novel techniques for testing and characterization of nanomaterials.

Bei Peng Changhong Ke Yaling Liu Yong Zhu 

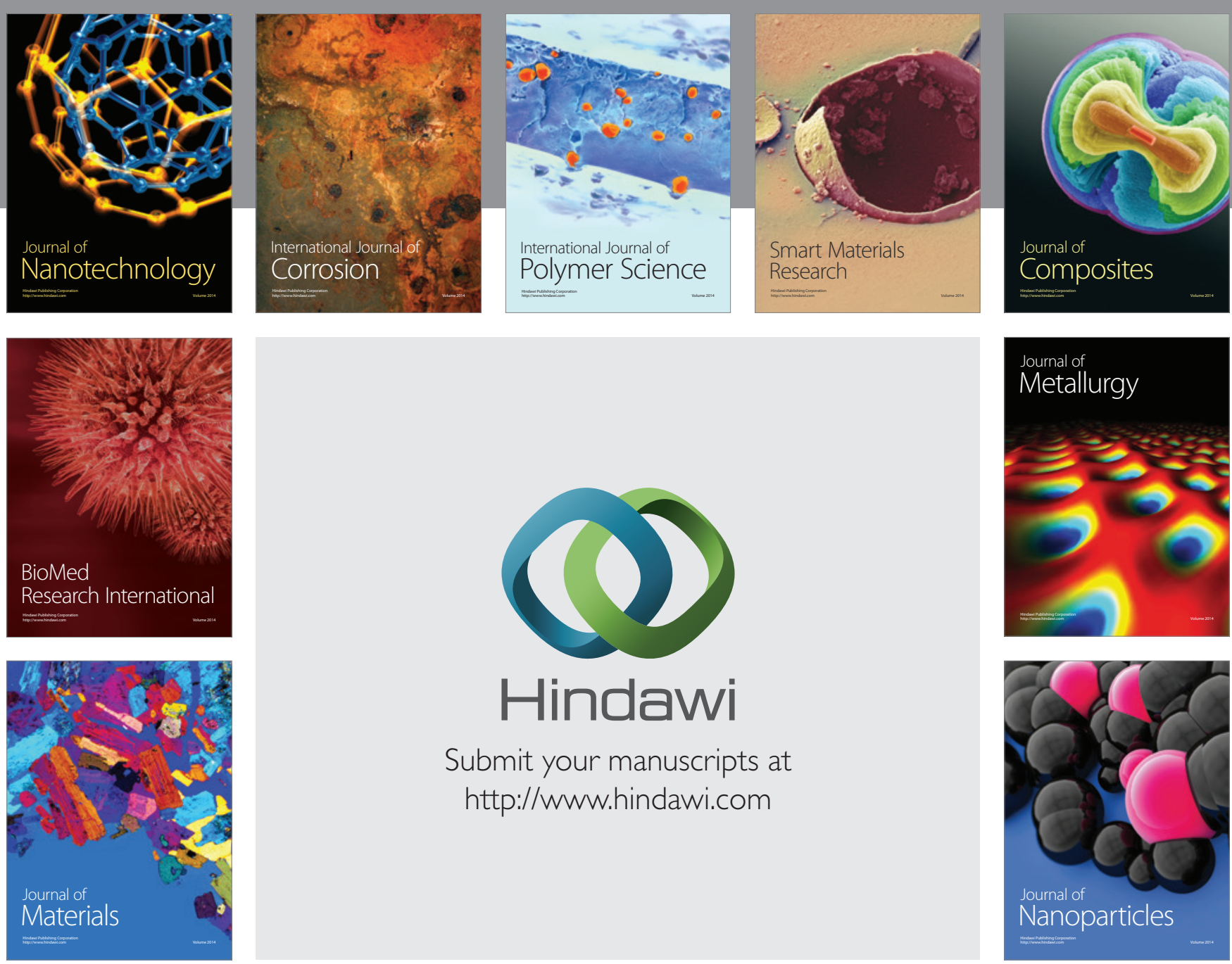

Submit your manuscripts at http://www.hindawi.com
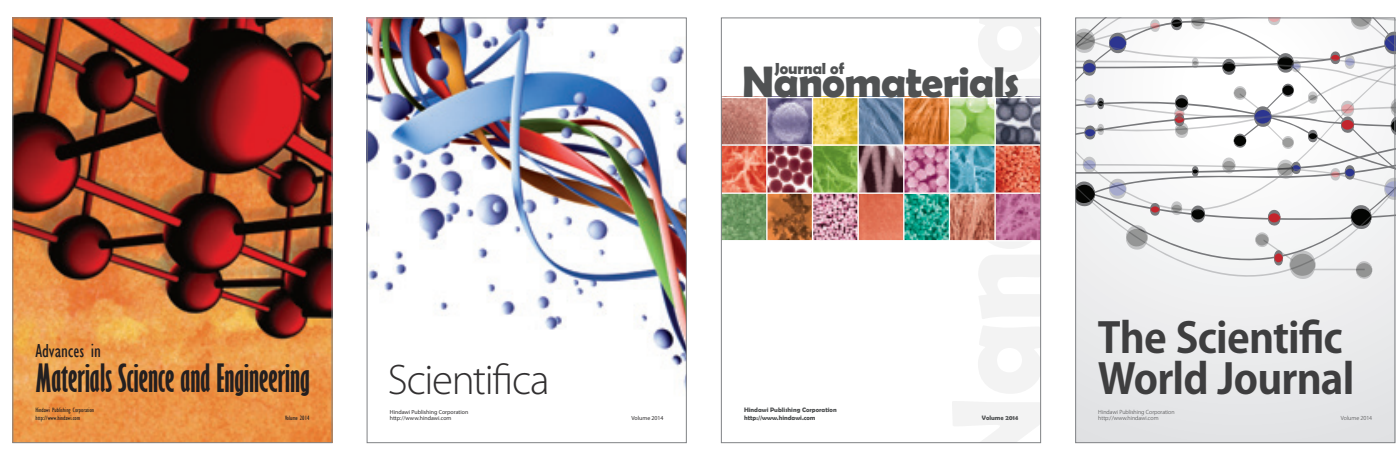

\section{The Scientific World Journal}
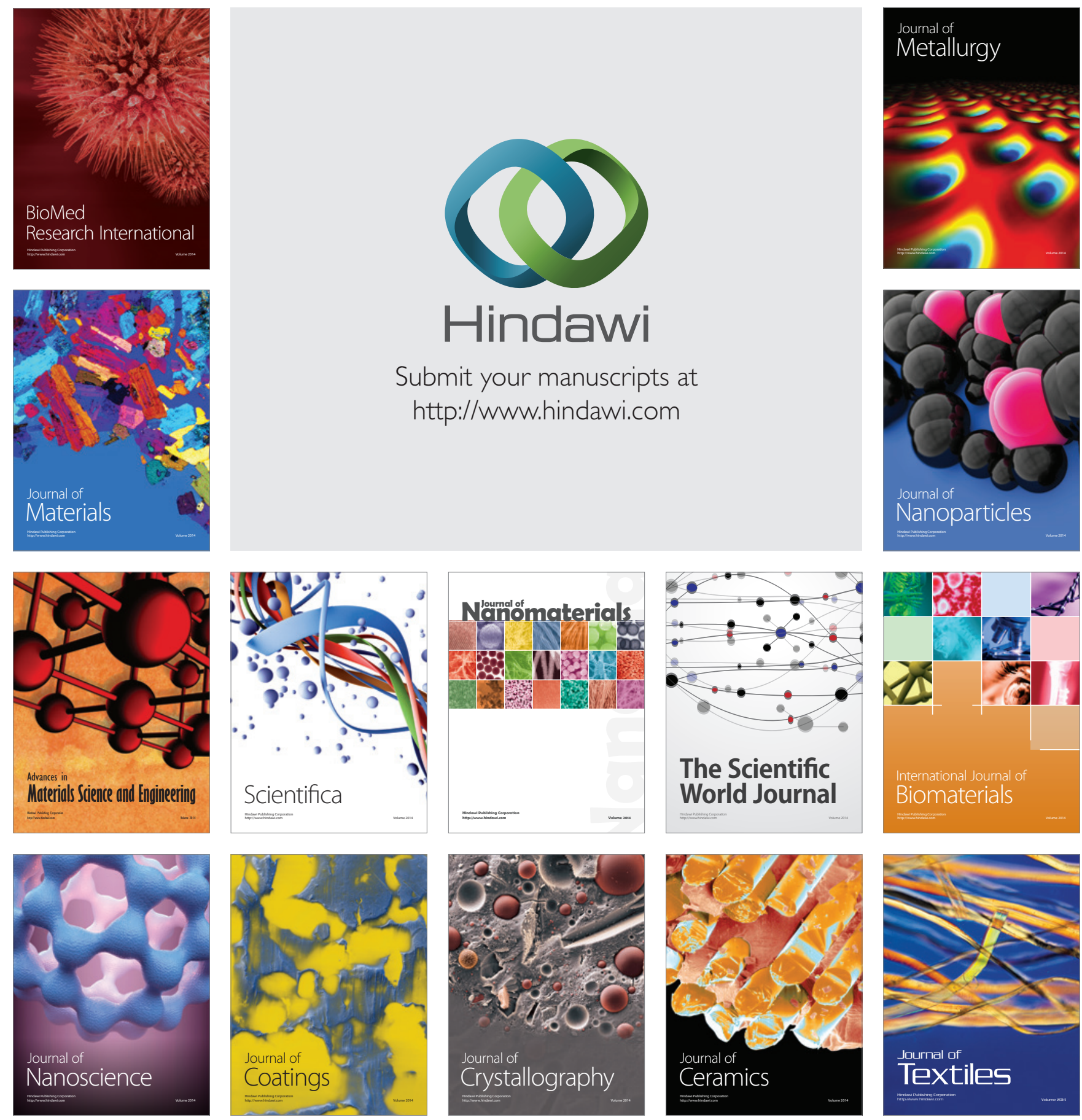